\title{
Good vs. Poor Self-rated Diabetes Control: Differences in Cardiovascular Risk and Self-care Activities
}

Authors

Affiliations
K. J. Smith ${ }^{1,2}$, R. Rabasa-Lhoret ${ }^{3,4,5}$, I. Strychar ${ }^{4,5,6}$, A. D. Karelis ${ }^{3,7}$, M. Clyde ${ }^{1,2}$, J. Levasseur ${ }^{3}$, C. Pinaroc ${ }^{3}$, M. Pedneault ${ }^{2}$, N. Schmitz ${ }^{1,2,4,8}$

Affiliation addresses are listed at the end of the article
Key words

- diabetes

- self-care

- HbA1c

- blood pressure

- cholesterol received $\quad 16.10 .2013$

first decision 20.12.2013

accepted $\quad$ 15.01.2014

Bibliography

DOI http://dx.doi.org/

10.1055/s-0034-1367005

Published online:

March 12, 2014

Exp Clin Endocrinol Diabetes

2014; 122: 236-239

(C) J. A. Barth Verlag in

Georg Thieme Verlag KG

Stuttgart · New York

ISSN 0947-7349

Correspondence

K. J. Smith, PhD

Douglas Mental Health

University Institute

McGill University

6875 LaSalle Boulevard

$\mathrm{H} 4 \mathrm{H} 1 \mathrm{R} 3$

Montreal, Quebec

Canada

Tel.: + 1/514/761 6131

Ext: 3334

Fax: +1/514/888 4064

kimberly.smith@douglas.

mcgill.ca

\section{Abstract}

$\nabla$

Aim: The aim of this study was to assess differences in cardiovascular risk and performance of self-care activities in people who rated their diabetes control as good or poor.

Methods: A sub-sample of 77 participants who took part in the Evaluation of Diabetes Treatment telephone interview were invited into a clinic to complete a series of laboratory examinations. Self-rated diabetes control was validated using the following laboratory markers: HbA1c, total cholesterol/HDL cholesterol ratio and LDL cholesterol. Differences in blood pressure and BMI were also assessed. Finally, all participants also com-

\section{Introduction}

$\nabla$

The identification of pertinent single-item selfrated screening questions that capture the multidimensional nature of health and disease is becoming increasingly important. These singleitem questions can act as indicators of health status in health surveys and be used as screening questions for clinicians.

Self-rated diabetes control is a single-item question which has the potential to be used as an indicator of diabetes management in both research and clinical settings. Results from previous work indicate that this question is associated with diabetes outcomes, self-care, psychological status and HbA1c (Lange \& Piette 2005, Smith et al. 2013, Smith et al. 2012). However, to our knowledge no study has undertaken a clinical assessment of this question within the framework of diabetes management guidelines.

People with diabetes are recommended to manage their diabetes via a regimen of healthful eating, physical activity, smoking cessation, weight control and monitoring for complications (International Diabetes Federation 2013) in order to pleted the Summary of Self-Care activities questionnaire.

Results: Those people who rated their diabetes control as fair or poor had a significantly higher BMI, HbA1c levels, total cholesterol/HDL-cholesterol ratio and systolic blood pressure. When asked about self-care activities in the past week, those people who reported their diabetes control was fair/poor had spent significantly fewer days following a general diet and exercising.

Conclusions: People with poor self-rated diabetes control have unfavourable cardiovascular risk and decreased performance of self-care activities.

decrease cardiovascular risk and avoid complications.

The aim of this study was to examine differences in cardiovascular risk and adherence to self-care recommendations in those people who rate their diabetes control as good or poor.

\section{Methods \\ $\nabla$}

\section{Participants}

All participants completed the baseline telephone interview of the Evaluation of Diabetes Treatment (EDIT) Study. The EDIT study sampled 2028 people in the Quebec community with diabetes. Inclusion criteria were: diagnosis of type 2 diabetes mellitus for $\leq 10$ years, being insulinnaïve and aged 40-75 (more details see (Smith et al. 2013).

The EDIT clinical sub-study was conducted at the Instituts de Recherches Cliniques de Montréal (IRCM). People who expressed interest in the study during the telephone interview and lived within a $30 \mathrm{~km}$ radius of the IRCM (total 279 people) were invited to participate. A total of 92 peo- 
ple agreed to participate, with 79 people completing the study ( $\odot$ Table 1 ) and 77 answering the question pertaining to diabetes control.

All participants gave their informed consent prior to undertaking the study and upon study completion were given a $\$ 15.00$ cheque and results of their clinical tests. The study was conducted in line with Declaration of Helsinki guidelines, and given ethical approval by the IRCM ethics committee.

\section{Self-rated Diabetes Control}

Participants were asked to rate their diabetes control within the previous month on a 5-point likert scale running from excellent to poor. Respondents were dichotomised into 2 groups: Good (responded excellent/very good/good) and poor (responded fair/ poor) in line with previous work (Smith et al. 2013, Smith et al. 2012).

\section{Measurement of cardiovascular risk factors}

All participants had their weight $(\mathrm{kg})$ and height $(\mathrm{cm})$ measured by a research nurse. BMI was calculated as $\mathrm{kg} / \mathrm{m}^{2}$.

Sitting blood pressure (BP) was determined by an automatic sphygmomanometer machine (Welch Allyn).

Fasting blood samples were collected from all participants. Serum concentrations of total cholesterol (TC), HDL-cholesterol, LDL-cholesterol and HbA1c were measured using the COBAS INEGRA 400 (Roche Diagnostic, Canada). For more details on laboratory measures see Lavoie et al. (Lavoie et al. 2012).

\section{Measurement of self-care activities}

Participants were asked if they were a current smoker with never or former smokers being classified as non-smokers.

Participants were also administered the summary of self-care activities questionnaire (Toobert et al. 2000) which asks on how many of the last 7 days participants spent adhering to diabetesspecific self-care activities including diet (specific diet which assessed consumption of certain foods and general diet which assessed the diet as a whole), exercise, foot care, medication adherence and blood glucose monitoring recommendations. All scores were averaged across each dimension so that a composite score for days within a week spent adhering to recommendations was obtained. Smoking was categorised according to smoking status (current smoker vs. non-smoker).

\section{Socio-demographic and diabetes characteristics}

All participants completed a medical history interview comprising of questions on socio-demographic characteristics, date of diabetes diagnosis and diagnoses of diabetes complications (cardiovascular disease, nephropathy, and neuropathy).

\section{Statistics}

Data were assessed using independent-samples t-tests and crosstabulations.

\section{Results}

$\nabla$

In total 65 people reported having good (excellent/very good/ good) control and 12 reported having poor (fair/poor control). There were no significant differences between groups for any sociodemographic or diabetes characteristics other than age ( $\odot$ Table 1). Those people who reported poor control had significantly higher mean BMI, HbA1c, TC/HDL-cholesterol ratio and systolic BP ( $\bullet$ Table 1). There were no significant betweengroup differences for either LDL-cholesterol or diastolic BP. When assessed using the summary of self-care activities, those people reporting poor control reported significantly fewer days spent adhering to general diet and exercise recommendations (○ Table 1). However, there were no significant differences between-groups for specific diet, blood glucose testing, foot care, medication adherence or the likelihood of being a current smoker ( 0 Table 1 ).

\section{Discussion}

\section{$\nabla$}

Results from this study indicate that those people who rate their diabetes control as poor have a significantly and clinically worsened cardiovascular risk profile and spend significantly fewer days adhering to self-care recommendations.

To our knowledge this is the first study that has sought to ascertain the clinical relevance of self-rated diabetes control by investigating the association of response to this question within diabetes management guidelines. In Canada people with diabetes are taught to be aware and mindful of their "ABC" (Association 2013): $\mathrm{HbA} 1 \mathrm{c} \leq 7 \%, \mathrm{BP}<130 / 80 \mathrm{~mm} \mathrm{Hg}$, LDL-cholesterol $<2.0 \mathrm{mmol} / \mathrm{L}$ and TC/HDL-cholesterol ratio<4. Our results indicate that people reporting poor control had higher average values for all components of their $\mathrm{ABC}$ than those recommended. Furthermore, people reporting poor diabetes control had significantly higher HbA1c, TC/HDL-cholesterol ratio, systolic BP and BMI. These observations indicate self-rated diabetes control may have clinical relevance to unmask cardiovascular risk.

People reporting poor control also described spending significantly less time adhering to diet and exercise self-care recommendations; both key components of diabetes self-care and replications of our previous findings (Smith et al. 2013, Smith et al. 2012).

There were no significant differences between-groups for medication adherence, glucose monitoring, foot care or the likelihood of being a current smoker.

Results from this study provide further evidence that self-rated diabetes control may be a single-item question that provides validity across a wide range of indicators of self-care in people with diabetes. This indicates that the question may have clinical validity as a screening question in order for clinicians to ascertain whether more specific tests for cardiovascular risk and additional questions about self-care may be necessitated. The results also indicate that the question may be used as a proxy for HbA1c in interview-based surveys in where it may not be feasible to collect clinical data.

The combination of factors that are worsened in people reporting poor diabetes control are also risk factors for the development of diabetes complications such as cardiovascular disease (Stamler et al. 1993). Thus, there is a need for future research to determine if this self-rated outcome can act as a predictor for morbidity and mortality in people with diabetes. There is also a need for large-scale future research to replicate this result and assess the validity of this question within clinical practice.

The strengths of this study are that participants were sampled from a larger representative, homogenous community-based sample (Smith et al. 2013). However, generalizability of data is limited by specific inclusion criteria and inferences on direction of causality are limited by cross-sectional data. We were also unable to control for important confounders such as age and sex 


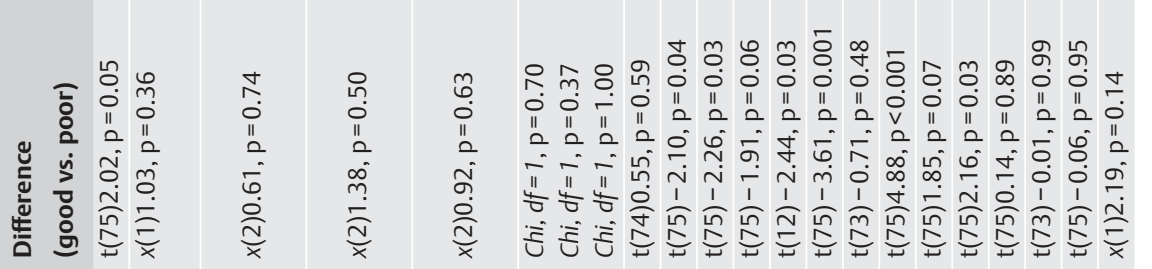

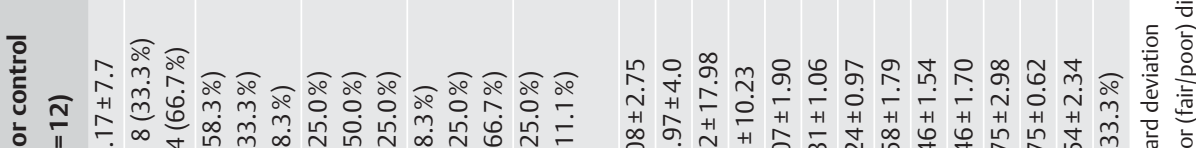

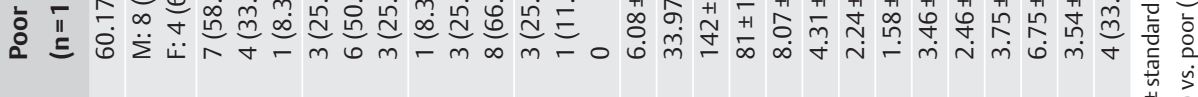

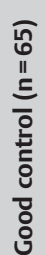

๑ே ๑

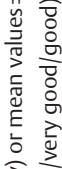

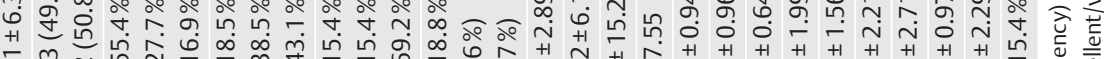

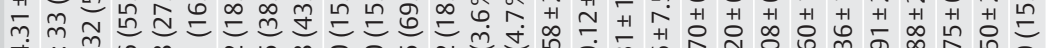

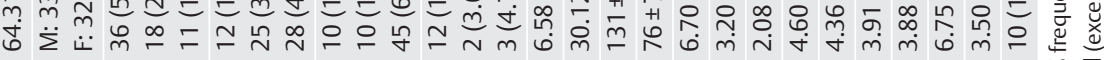

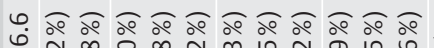

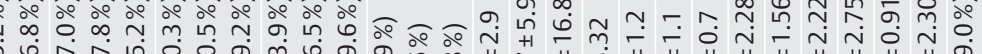

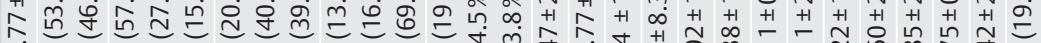

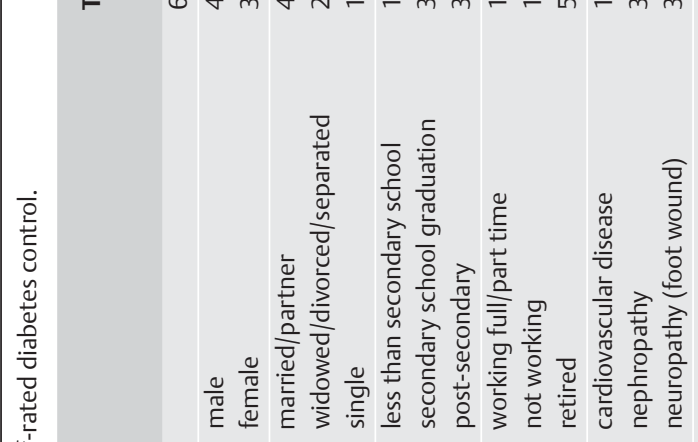

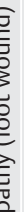

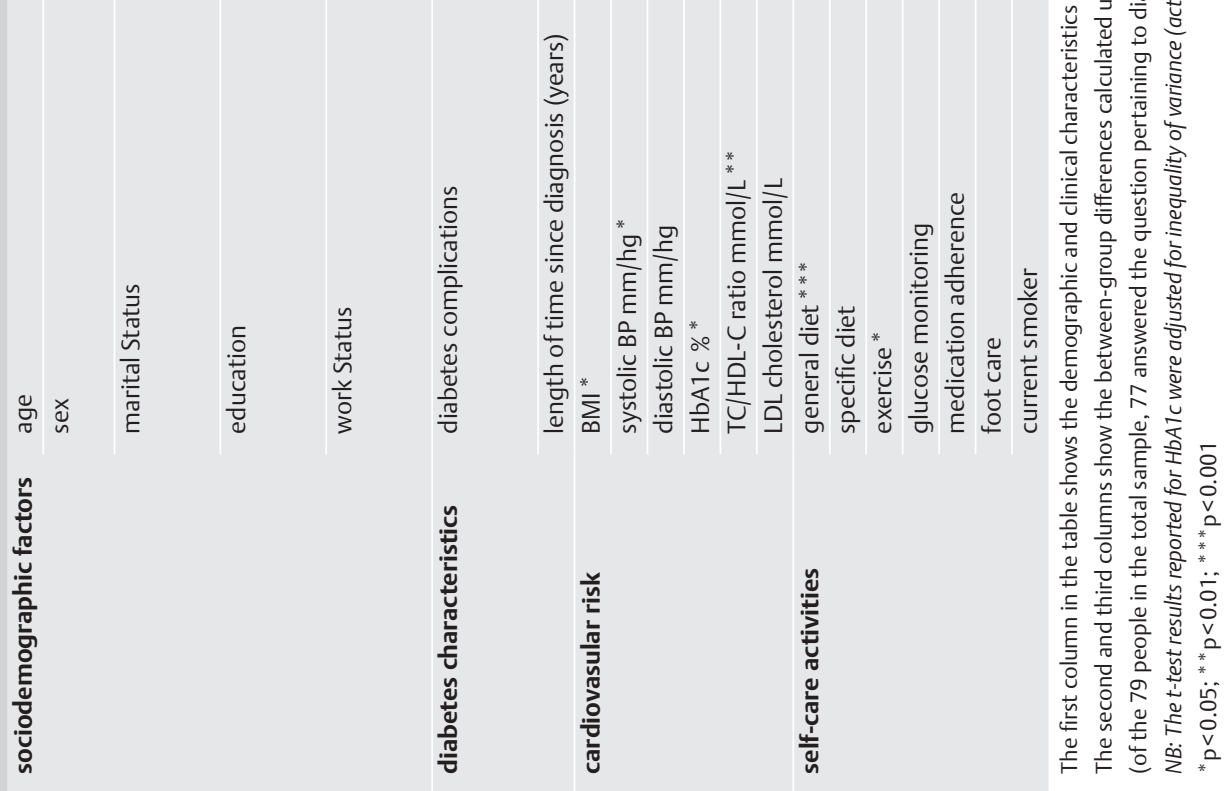


in our analysis due to the impact this would have on the study power. However, these are important confounders that have been controlled for in our previous larger studies (Smith et al. 2012, 2013).

Overall these findings indicate that people with poor self-rated diabetes control have unfavourable cardiovascular risk and decreased adherence to self-care activities.

\section{Author Contributors}

$\nabla$

The clinical study was designed by NS, IS, RRL, AK and KS. The data was collected by JL, CP, MC, MP and KS. The data analysis and manuscript were prepared by KS. All authors critically reviewed the article and gave final approval for this version to be published.

\section{Funding Sources}

This research and the corresponding author were funded by an operating grant from the CIHR. RRL, NS and AK hold scholarships from the FRSQ.

\section{Acknowledgements}

This research was funded by an operating grant from the Canadian Institutes of Health Research. We would also like to thank all the EDIT participants who kindly gave up their time to help us conduct this study. We would also like to thank all the staff at the IRCM and CHUM-Hotel-Dieu who helped with this project.

Conflict of interest: All authors disclose that they have no conflicts of interest.
Affiliations

${ }^{1}$ Department of Psychiatry, McGill University, Montreal, Quebec, Canada

${ }^{2}$ Douglas Mental Health University Institute, Montreal, Quebec, Canada

${ }^{3}$ Institut de Recherches Cliniques de Montréal, Montréal, Québec, Canada

${ }^{4}$ Montreal Diabetes Research Center, Montreal, Quebec, Canada

${ }^{5}$ Department of Nutrition, Faculty of medicine, Université de Montréal

${ }^{6}$ Research Center of the University of Montreal Hospital Center, Université de Montréal

${ }^{7}$ Department of Kinanthropology, UQAM, Montreal, Quebec, Canada

${ }^{8}$ Department of Epidemiology and Biostatistics, McGill University, Montreal, Quebec, Canada

\section{References}

1 Canadian Diabetes Association. Staying healthy with diabetes 2013; http://www.diabetes.ca/Files/StayHealthy.pdf

2 International Diabetes Federation. Management of Diabetes 2013; http://www.idf.org/treatment-diabetes

3 Lange LJ, Piette JD. Perceived health status and perceived diabetes control: psychological indicators and accuracy. J Psychosom Res 2005; 58: $129-137$

4 Lavoie ME, Faraj M, Strychar I et al. Synergistic associations of physical activity and diet quality on cardiometabolic risk factors in overweight and obese postmenopausal women. Br J Nutr 2012; May 9: 1-10

5 Smith KJ, Gariepy G, Pedneault $M$ et al. Exploring the association of psychological status with self-rated diabetes control: results from the montreal evaluation of diabetes treatment study. Psychosomatics 2013; 54: 35-43

6 Smith KJ, Page V, Gariepy $G$ et al. Self-rated diabetes control in a Canadian population with type 2 diabetes: associations with health behaviours and outcomes. Diabetes Res Clin Pract 2012; 95: 162-168

7 Stamler J, Vaccaro 0, Neaton JD et al. Diabetes, other risk factors, and 12 -yr cardiovascular mortality for men screened in the Multiple Risk Factor Intervention Trial. Diabetes Care 1993; 16: 434-444

8 Toobert DJ, Hampson SE, Glasgow RE. The summary of diabetes selfcare activities measure: results from 7 studies and a revised scale. Diabetes Care 2000; 23: 943-950 